\title{
Associating the scale-up of insecticide-treated nets and use with the decline in all-cause child mortality in the Democratic Republic of Congo from 2005 to 2014
}

Johanna Karemere ${ }^{1,2^{*}}$, Ismael G. Nana ${ }^{1,2}$, Andrew Andrada ${ }^{1,2}$, Olivier Kakesa ${ }^{1,2}$, Eric Mukomena Sompwe ${ }^{3,4}$, Joris Likwela Losimba ${ }^{3,5}$, Jacques Emina ${ }^{6,7}$, Aboubacar Sadou ${ }^{8}$, Michael Humes ${ }^{8}$ and Yazoumé Yé ${ }^{1,2}$

\begin{abstract}
Background: To reduce the malaria burden and improve the socioeconomic status of its citizens, the Democratic Republic of Congo scaled up key malaria control interventions, especially insecticide-treated nets (ITNs), between 2005 and 2014. Since then, the effects of these interventions on malaria mortality and morbidity have not been assessed. This study aimed to measure the impact of the National Malaria Control Programme's efforts and to inform future control strategies.

Methods: The authors used data from the Demographic and Health Surveys 2007 and 2013-2014 to assess trends in all-cause childhood mortality (ACCM) against trends in coverage of malaria interventions at national and subnational levels. The authors used the plausibility argument to assess the impact of the malaria control interventions and used Kaplan-Meier survival probability and Cox proportional hazard models to examine the effect of ITN ownership on child survival. Contextual factor trends affecting child survival were also considered.

Results: Countrywide, household ownership of at least one ITN increased, from 9\% in 2007 to $70 \%$ in 2013-2014. All provinces experienced similar increases, with some greater than the national level. ITN use increased between 2007 and 2013-2014 among children under five (6\% to 55\%). Severe anaemia (haemoglobin concentration $<8 \mathrm{~g} / \mathrm{dl}$ ) prevalence among children aged 6-59 months significantly decreased, from 11\% (95\% confidence interval [CI] 9-13\%) in 2007 to $6 \%$ (95\% Cl 5-7\%) in 2013-2014. During the same period, ACCM declined, from 148 (95\% Cl 132-163) to 104 (95\% Cl 97-112) deaths per 1000 live births. The decline in ACCM was greater among children aged 6-23 months (relative reduction of $36 \%$ ), compared to children aged $24-59$ months (relative reduction of $12 \%$ ). Cox regression indicated that household ownership of at least one ITN reduced the risk of mortality by $24 \%$ among children under five (risk ratio $=0.76,95 \% \mathrm{Cl} 0.64-0.90$ ). Contextual factor analysis revealed marginal improvements in socioeconomic indicators and other health interventions.
\end{abstract}

*Correspondence: Johanna.Karemere@icf.com

${ }^{2}$ ICF, Rockville, MD, USA

Full list of author information is available at the end of the article

\section{$\triangle B M C$}

The Author(s) 2021. This article is licensed under a Creative Commons Attribution 4.0 International License, which permits use, sharing, adaptation, distribution and reproduction in any medium or format, as long as you give appropriate credit to the original author(s) and the source, provide a link to the Creative Commons licence, and indicate if changes were made. The images or other third party material in this article are included in the article's Creative Commons licence, unless indicated otherwise in a credit line to the material. If material is not included in the article's Creative Commons licence and your intended use is not permitted by statutory regulation or exceeds the permitted use, you will need to obtain permission directly from the copyright holder. To view a copy of this licence, visit http://creativeco mmons.org/licenses/by/4.0/. The Creative Commons Public Domain Dedication waiver (http://creativecommons.org/publicdomain/ zero/1.0/) applies to the data made available in this article, unless otherwise stated in a credit line to the data. 
Conclusions: Given the patterns of the coverage of malaria control interventions, patterns in ACCM by province, and marginal improvements in contextual factors, the authors conclude that the malaria control interventions have plausibly contributed to the decrease in ACCM in the Democratic Republic of Congo from 2005 to 2014.

Keywords: Malaria, Control, ITN, Kaplan-Meier, Cox proportional hazards, All-cause child mortality, Sub-Saharan Africa, DRC

\section{Background}

Over the last decade, malaria has been the leading cause of mortality and morbidity in the Democratic Republic of Congo (DRC), with an estimated $97 \%$ of the Congolese population at risk of malaria infection $[1,2]$. To improve the overall health of its citizens and reduce the socioeconomic burden of malaria, the country and its technical and financial partners invested significantly in proven malaria control interventions between 2005 and 2015. Using these funds, the National Malaria Control Programme (NMCP) set objectives to contribute to the elimination of malaria and reduce the malaria mortality and morbidity rates by $50 \%$ compared to the 2010 levels by 2015 [3]. The NMCP's strategy was to scale up and expand access to these key interventions: the use of insecticide-treated nets (ITNs), intermittent preventive treatment in pregnancy (IPTp), indoor residual spraying (IRS), and prompt and effective malaria case management $[3,4]$. DRC's efforts to achieve its malaria control goals focused on the scaleup of ITN coverage through free ITN mass distribution campaigns and routine distribution during antenatal care visits and expanded programme on immunization (EPI) visits (Fig. 1) [3].
The effects of scale up of malaria control intervention on all-cause child mortality (ACCM) have been studied in other countries in sub-Saharan Africa, however, no studies have been conducted in DRC $[5,6]$. This study aimed to fill this gap by using Demographic and Health Survey (DHS) data to assess the extent to which malaria control interventions have been implemented and scaled up, assess the trends of malaria morbidity and mortality from 2005 to 2014, and evaluate plausible association between interventions and the trends of the disease burden. The current national malaria strategic plan (NMSP) encompasses the period from 2016 to 2020, and the latest available DHS survey is from 2013 to 2014, which precludes the assessment of more recent trends $[2,7]$. The results of this assessment allows for a comparison between the intervention coverage achieved and the NMSP targets set for 2015 [3]. In addition, the results will be a key resource in providing baseline data on intervention coverage and malaria morbidity and mortality for the current 2016-2020 NMSP. Following the end of this NMSP, another evaluation can then be used to compare this baseline and evaluate the NMCP progress made on these key indicators [7].

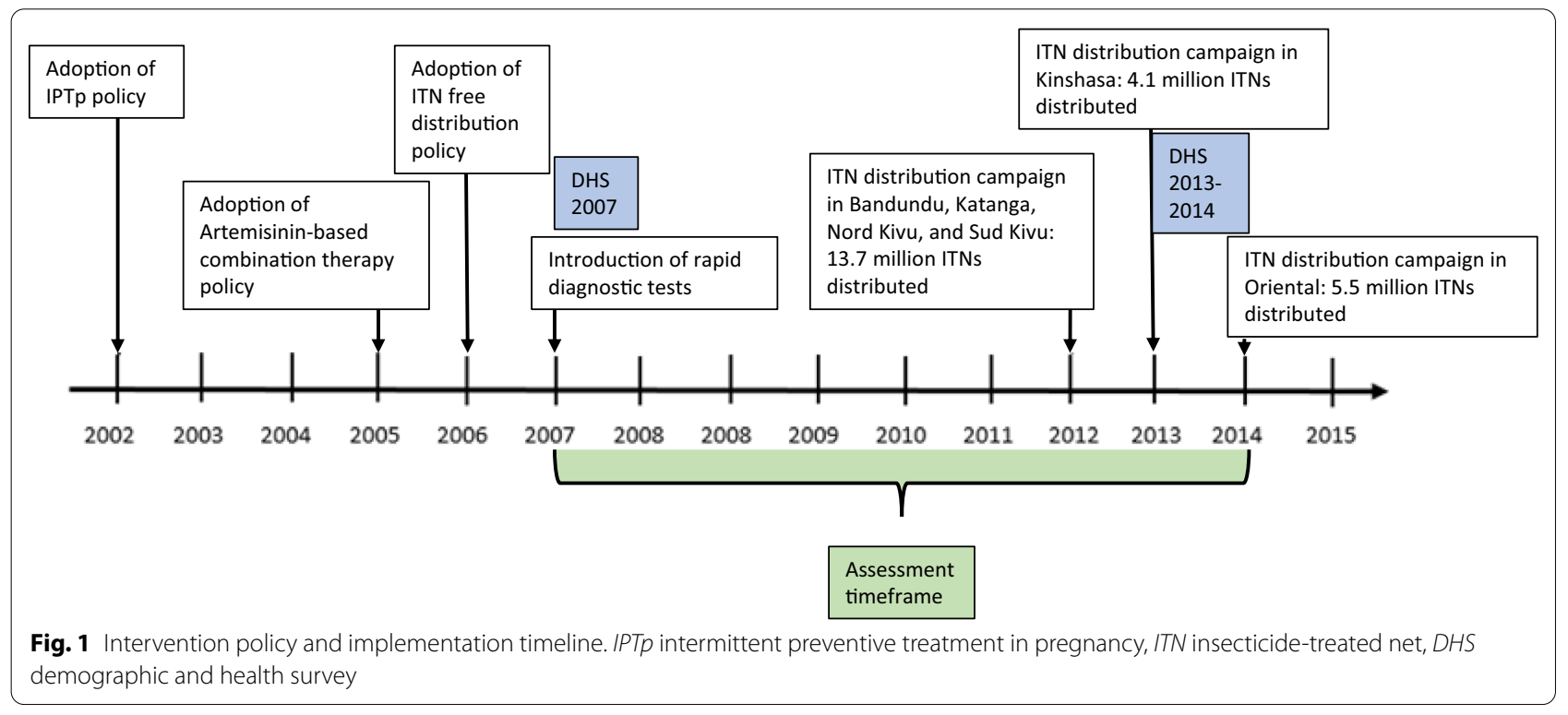




\section{Methods}

\section{Data sources}

This evaluation used nationally representative household survey data from the 2007 and 2013-2014 DHS [1, 2]. The authors omitted the 2010 and 2017-2018 DRC Multiple Indicator Cluster Survey from this study because of incongruous mortality estimate methodology. DHS calculates child mortality based on the birth history of women of childbearing age compared to the MICS, which calculates child mortality indirectly using the number of children born alive. Differences in sampling strategies may also affect interpretation of trends. An exploratory analysis of malaria indicators by region showed different trends across the surveys. In most cases, the MICS estimates were higher compared to the DHS, suggesting a sampling difference. The contextual factor data were obtained from the DHS. Gross domestic product per capita purchasing power parity, total annual rainfall, and temperature data were from the World Bank $[8,9]$.

\section{Study design}

This evaluation used the before-and-after plausibility approach as recommended by the Roll Back Malaria Partnership to End Malaria Monitoring and Evaluation Reference Group [10]. The rationale and use of this evaluation design to measure the primary impact of malaria control intervention scale-up on ACCM has been detailed in elsewhere $[5,11-13]$. Following previous research on the effectiveness of malaria control interventions, the authors hypothesized that improvement in coverage of these interventions (ITNs, IRS, IPTp, and case management) should result in a reduction in ACCM, assuming that other contextual factors (e.g., living conditions, nutritional status of children) have not substantially changed over the evaluation period [5]. The plausibility approach is strengthened by the simultaneous decline in other outcome measures such as malaria prevalence and severe anaemia concurrent with malaria control intervention scale-up. The use of ACCM as the primary impact indicator provides a robust measure that accounts for direct and indirect malaria mortality. In addition to the mortality indicator, morbidity was assessed through measuring trends in severe anaemia [haemoglobin $(\mathrm{Hb})$ concentration $<8 \mathrm{~g} / \mathrm{dl}$ ] prevalence among children aged 6-59 months from 2005 to 2013-2014 [14]. Data on the prevalence of malaria among children aged 6-59 months were not available for the DHS 2007; therefore, it was not possible to assess malaria infection trends during the evaluation period.

\section{Analytical approach \\ Trends analysis}

Descriptive analysis was conducted using Stata version 14 (Stata Corporation, College Station, TX). To account for the hierarchical design, the SVY commands were used, with household weights considered for each analysis. Percentages and rates were calculated with $95 \%$ confidence intervals (CIs) to assess trends between 2007 and 2013-2014 at the national and subnational levels. The 11 provinces that constituted DRC until 2015 were examined. The analysis encompasses changes in intervention coverage, including household ownership and use of ITNs and access to malaria diagnosis and treatment for children with fever, but also changes in outcome measures such as severe anaemia and ACCM. Key household survey indicators used for this evaluation are provided in Table 1 [15].

\section{Regression analysis}

The regression analysis included Kaplan-Meier survival and Cox proportional hazard models to support the plausibility argument. The Kaplan-Meier survival analysis used the full birth history data from the DHS 2013-2014, which were transformed into a 10-year retrospective

Table 1 Key household surveys indicators used for this evaluation [15]

\begin{tabular}{ll}
\hline Indicator & Definition \\
\hline $\begin{array}{l}\text { Vector control indicators } \\
\text { ITNs }\end{array}$ & $\begin{array}{l}\text { Proportion of households with at least one ITN } \\
\text { Proportion of households with at least one ITN for every two people } \\
\end{array}$ \\
$\begin{array}{l}\text { Pase management indicators } \\
\text { Early access to diagnosis and effective } \\
\text { treatment }\end{array}$ & $\begin{array}{l}\text { Proportion of children under } 5 \text { years old with fever in last } 2 \text { weeks who had a finger or heel stick } \\
\text { Impact indicators } \\
\text { Morbidity } \\
\text { Mortality }\end{array}$ \\
\hline
\end{tabular}


(2004-2013) longitudinal dataset reflecting individual child observations from birth until the date of the survey or, in the unfortunate event, the death of the child. Kaplan-Meier survival estimates were calculated, and comparisons were made for survival probability of children aged 0-59 months before (2004-2008) and after (2009-2013) the expansion of malaria control interventions. The assumption is that child survival would improve post-intervention scale-up, compared to preintervention scale-up. The outcome variable was defined as the age at which a child dies or the age at interview for those who survived. A dichotomous variable (coded 1 if the child died and 0 if the child was alive) was used to define the censoring status.

Cox proportional hazards regression used the same longitudinal birth history dataset as the Kaplan-Meier analysis. This model allows for use of time-varying covariates to produce a hazard ratio [16-18]. When the hazard ratio is greater than one, there is an increased risk of mortality in the corresponding category compared to the reference category. Conversely, the risk of dying is lower when the hazard ratio is less than one. The hazard rate in the Cox model is computed as:

$$
h\left(\frac{t}{z j}\right)=h_{0}(t), \exp (\beta j z j(t)),
$$

where the regression coefficients are to be estimated from the data. The term $h_{0}(\mathrm{t})$ is the baseline hazard function (the hazard when $\mathrm{z}=0), \mathrm{zj}(\mathrm{t})$ is the individual covariates vector, and $\beta j$ is a vector of the regression parameters that indicates the effects of these covariates, some of them varying with $\mathrm{t}$ (hence, the term time-varying covariate). The relative hazards are given by $\exp (\beta j z j(t)$.

To identify an individual child's exposure to an ITN, data on the duration of ownership of ITNs were used to construct a time-varying variable of ITN ownership for up to 2 years before the survey (2011-2013). The model included all children aged 0-59 months for the 2-year period of exposure to ITN ownership to account for recall bias related to duration of ITN ownership. The analysis time was defined in months from the beginning of the period, allowing the introduction of age as a covariate in the analysis. Each child was observed from the beginning of the observation period until censoring due to death or until the date of the survey. A dichotomous variable (coded 1 if the child died and 0 if the child was alive) was used to define the censoring status. The Cox proportional hazards model assessed the relationship between household ITN ownership and child mortality (deaths of children aged 0-59 months) over the 24 months preceding the survey in each malaria-endemic zone. The model was adjusted for child's age (month), child's sex, mother's age (year) at child's birth, mother's education, parity (number of children ever born), household wealth quintiles, and place of residence (urban/rural) because these co-variates are likely to be associated with both mortality and household ownership of ITN.

\section{Results \\ Trend in intervention coverage Ownership of ITNs}

At the national level, the proportion of households owning at least one ITN increased significantly by 61 percentage points, from 9\% (95\% CI 8.0-10.6\%) in 2007 to $70 \%$ (95\% CI 68.6-71.4\%) in 2013. Similar patterns were observed in all the provinces, with percentage point increases higher than the national increase in Equateur (79 percentage points), Bandundu (76 percentage points), Katanga (72 percentage points), and Sud-Kivu (64 percentage points) (Fig. 2).

\section{Use of ITNs among children under five}

At the national level, the use of ITNs in children under 5 years of age in all households significantly increased, from 6\% (95\% CI 5-7\%) in 2007 to 55\% (95\% CI 54-57\%) in 2013. The highest increases were observed in the provinces of Bandundu (73 percentage points), Equateur (63 percentage points), Katanga (55 percentage points), and Sud-Kivu (55 percentage points) (Fig. 3).

\section{Use of recommended malaria treatment among children under five}

The proportion of children under five with fever in the 2 weeks preceding the survey treated with artemisininbased combination therapy (ACT) slightly improved from 2007, with an increase from less than $1 \%$ in 2007 to $5 \%$ in 2013 (Fig. 4). Subnationally, the use of ACT also increased, with improvements in every province. However, access to ACT remained very low, with $5 \%$ of children with fever treated with ACT nationally.

\section{Change in morbidity and mortality Morbidity: change in severe anaemia}

At the national level, the prevalence of severe anaemia in children decreased significantly, from 11\% (95\% CI $9-13 \%$ ) in 2007 to $6 \%$ (95\% CI 5-7\%) in 2013. At the subnational level, a significant decrease in the prevalence of severe anaemia among children was observed in the provinces of Equateur, from 16\% (95\% CI 10-24\%) in 2007 to $5 \%(95 \%$ CI 3-8\%) in 2013, and Oriental, from $16 \%(95 \%$ CI $10-25 \%)$ in 2007 to $8 \%(95 \%$ CI $5-13 \%)$ in 2013 (Fig. 5). The national prevalence of severe anaemia compared with intervention coverage matches an increase in coverage of malaria intervention (Fig. 6). 


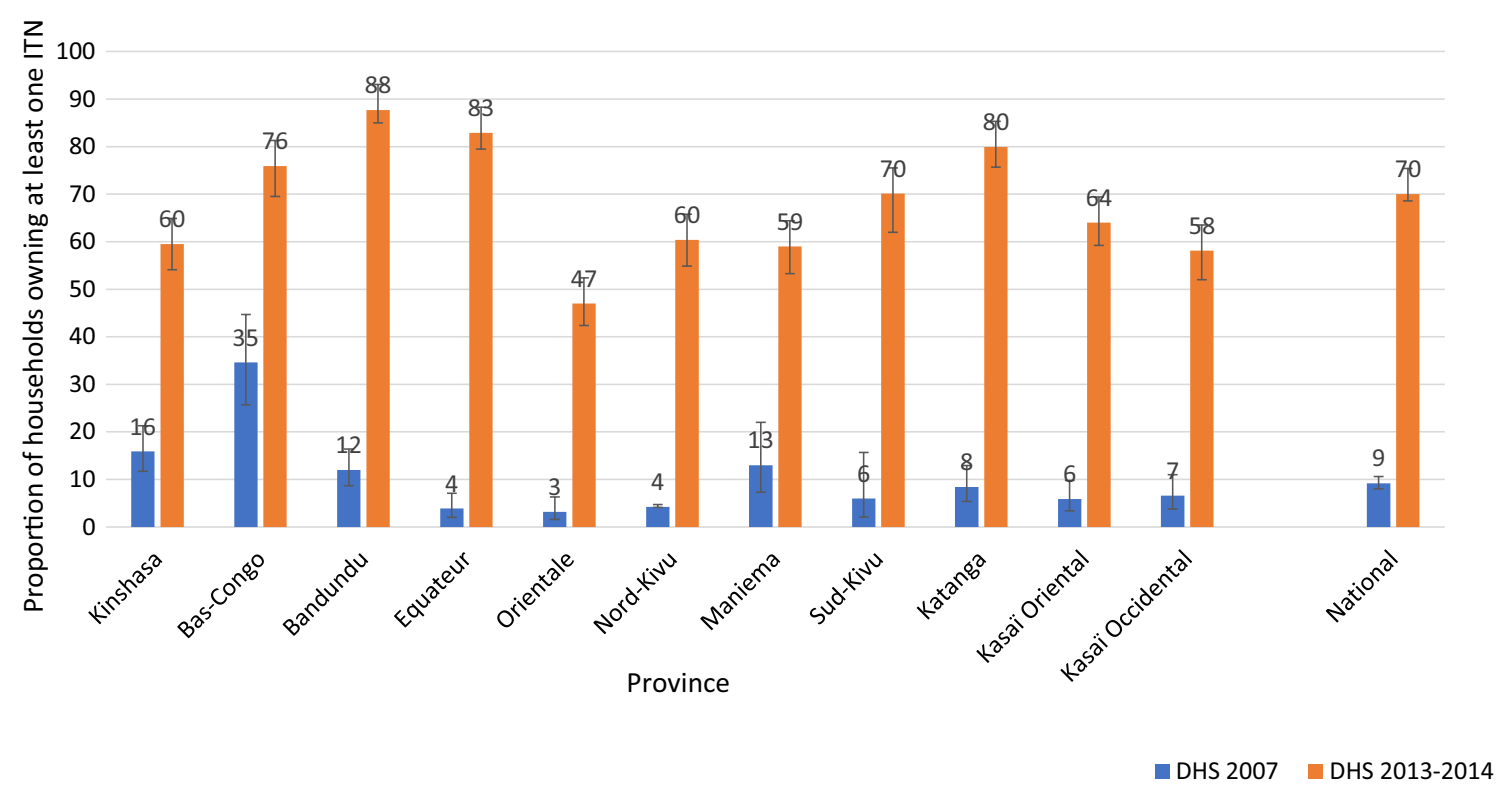

Fig. 2 Evolution of the proportion of households with at least one ITN at the national level and by province, in the DRC from 2007 to 2013 . ITN insecticide-treated net

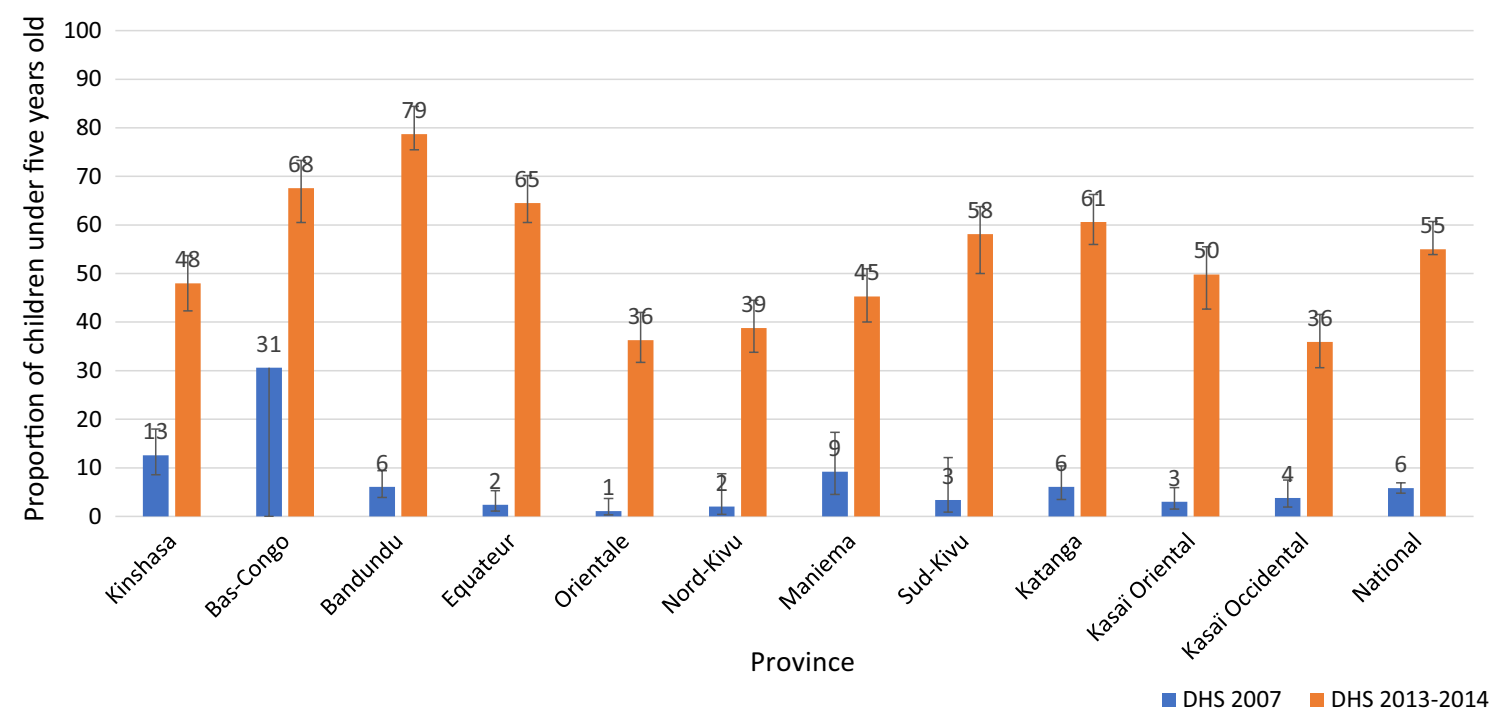

Fig. 3 Evolution of proportion of children under 5 years old who slept under an ITN the previous night at the national level and by province, in the DRC from 2007 to 2013. ITN insecticide-treated net

\section{Mortality: change in ACCM}

The ACCM rate significantly declined between 2007 and 2013-2014 by 30\%, from 148 (95\% CI 133-163) per 1000 live births in 2007 to 104 (95\% CI 98-111) per 1000 live births in 2013. By region, the largest decreases (relative reductions ranging from 15 to $52 \%$ ) were found in
Bas-Congo, Bandundu, Maniema, and Oriental provinces (Fig. 7). The observed decrease of ACCM is inversely proportional to the increase in ownership and use of ITNs (Fig. 6). 


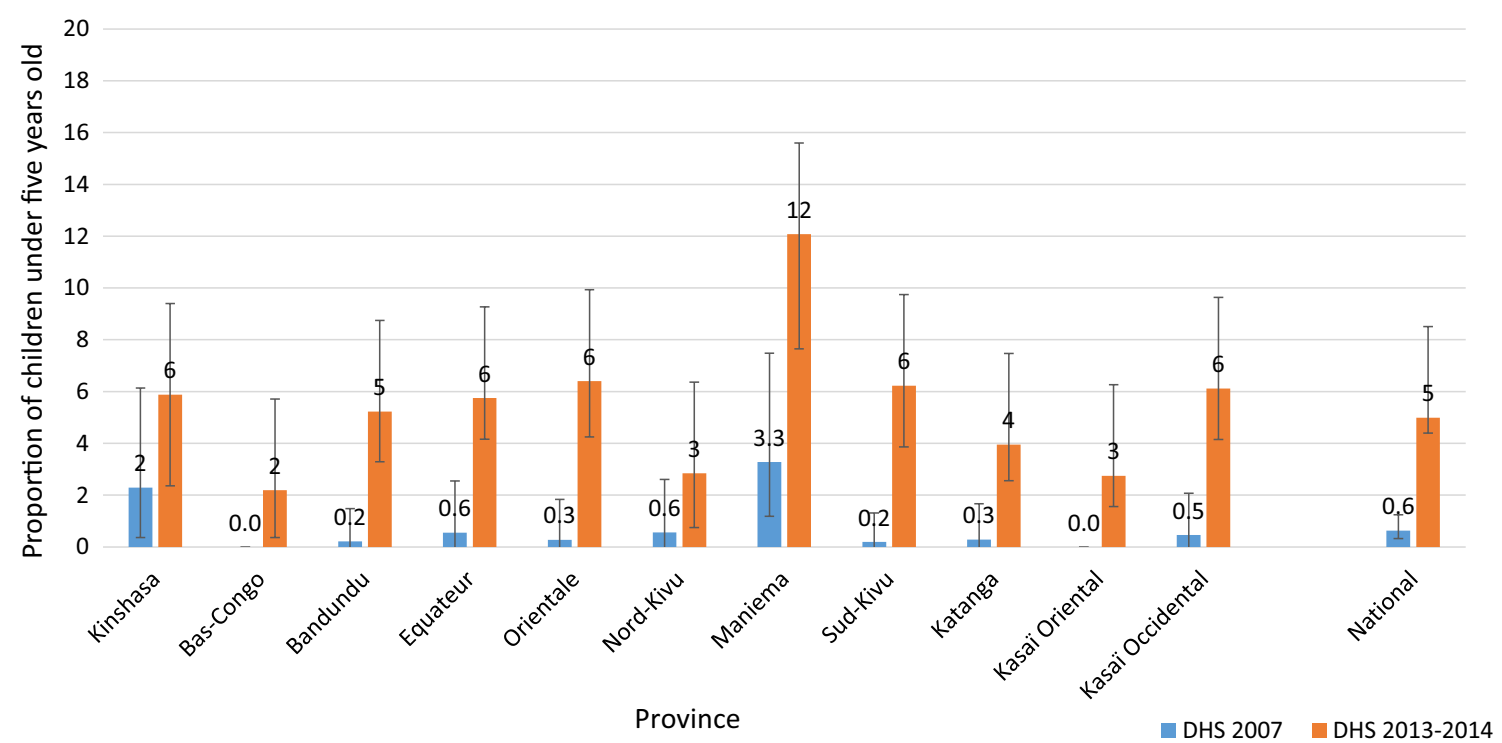

Fig. 4 Evolution of the proportion of children under five with fever treated with an ACT the national level and by province, in the DRC from 2007 to 2013. ACT artemisinin-based combination therapy

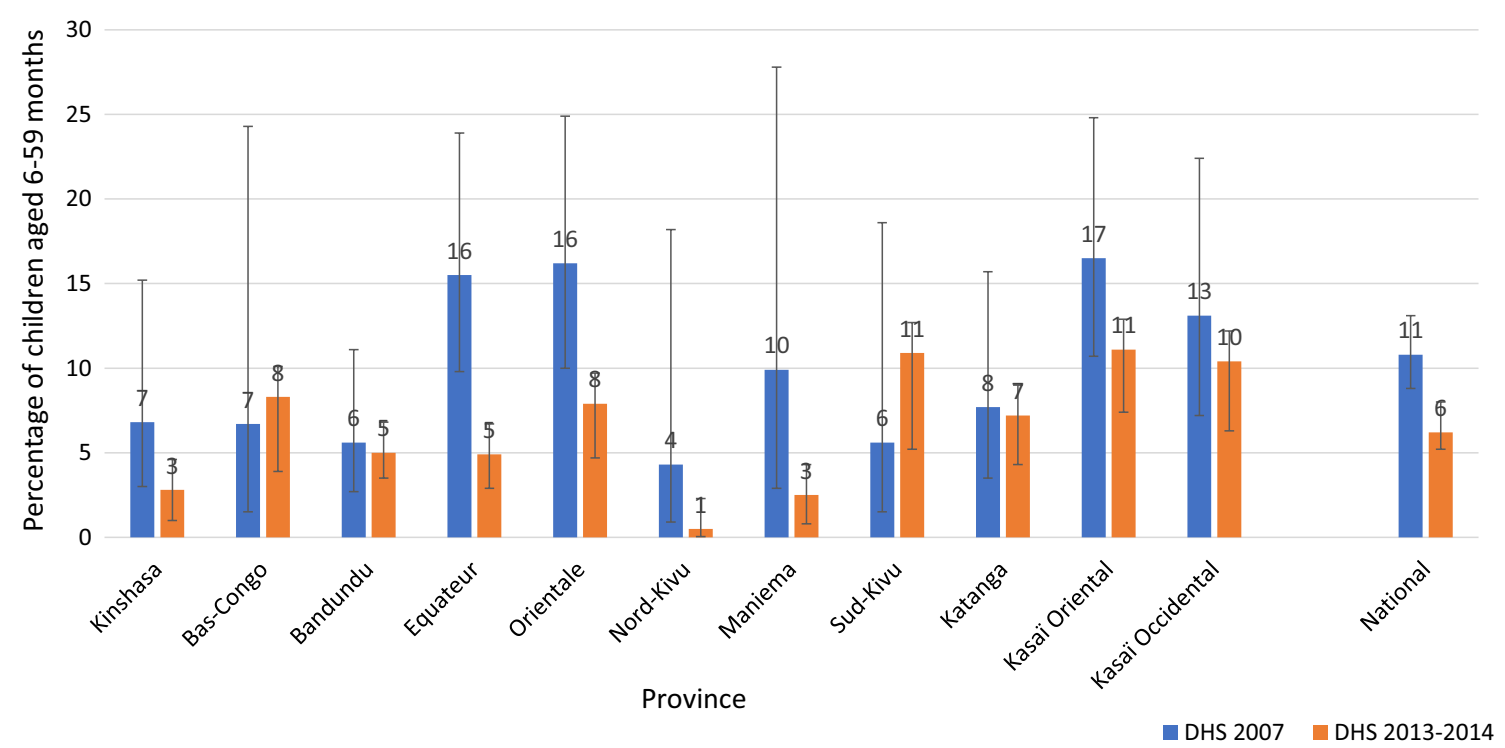

Fig. 5 Prevalence of severe anaemia $(\mathrm{Hb}<8 \mathrm{~g} / \mathrm{dl})$ among children aged 6-59 months by province, in the DRC from 2007 to 2013 . Hb haemoglobin

\section{Change in mortality risk associated with scale-up of ITN ownership \\ Kaplan-Meier survival probability}

Kaplan-Meier survival probability analysis shows a significant improvement in survival probability of children aged 0-59 months between 2004 and 2013-2014.
The survival probability was significantly higher among children born between 2009 and 2013, the period corresponding to the scale-up of malaria control interventions, compared to children born between 2004 and 2008, when the coverage of interventions was still low 


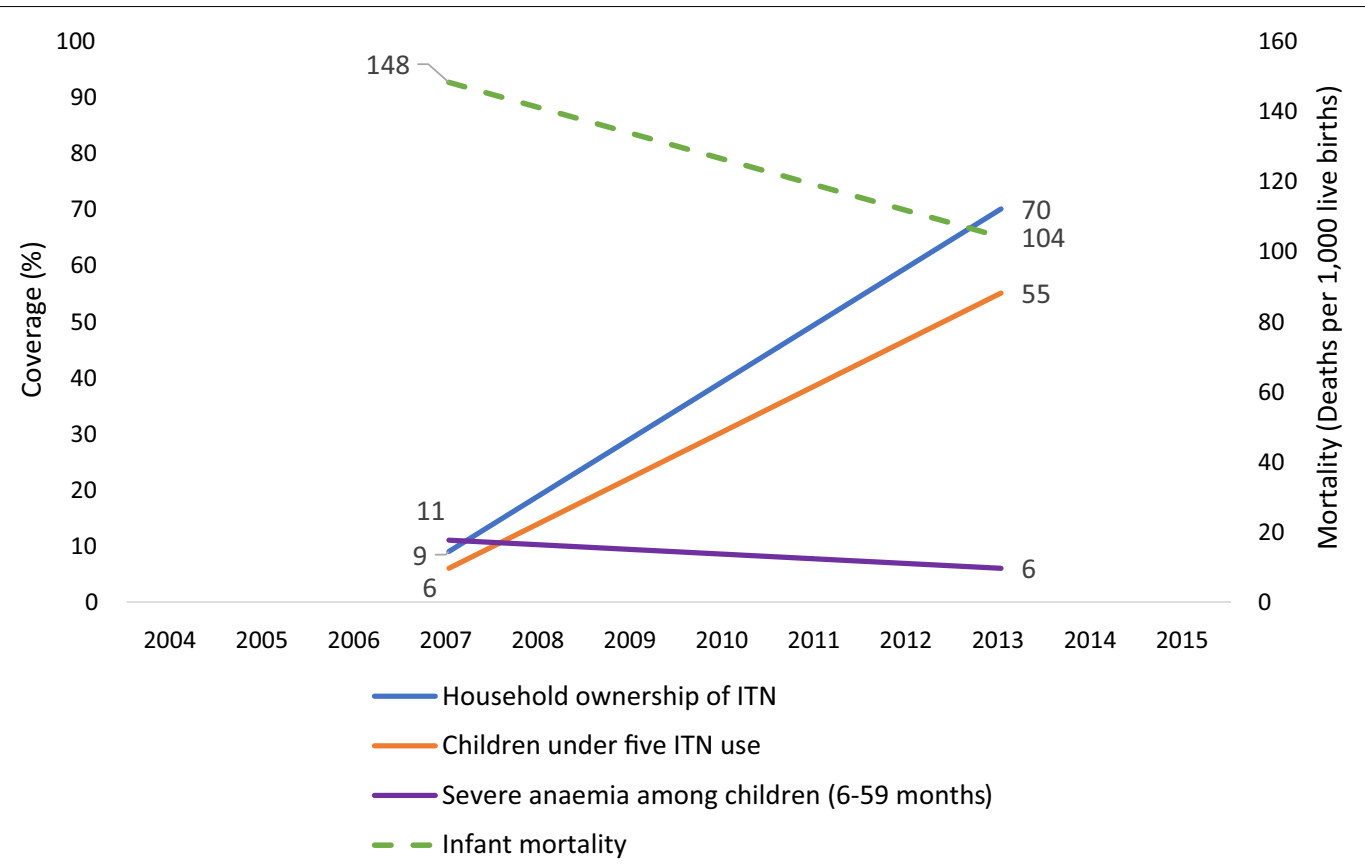

Fig. 6 Summary of trends in intervention coverage, severe anaemia, and malaria mortality in children under five, in the DRC from 2007 to 2013 . ITN insecticide-treated net

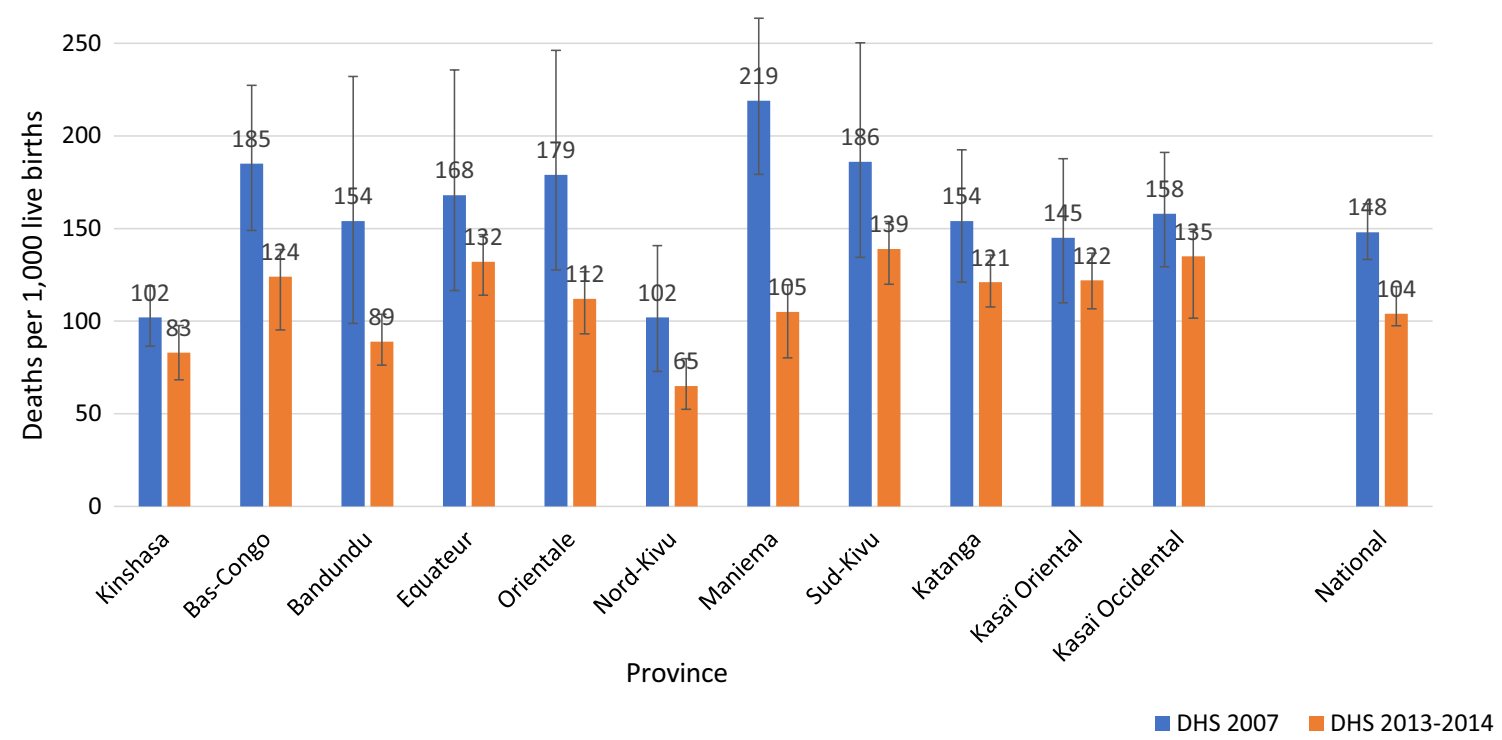

Fig. 7 Trends in all-cause mortality among children under five by province, in the DRC from 2007 to 2013

(Fig. 8). Among children aged 24-36 months, the probability of survival among children in 2004-2008 was approximately $89 \%$, compared to $92 \%$ among children in 2009-2013. 


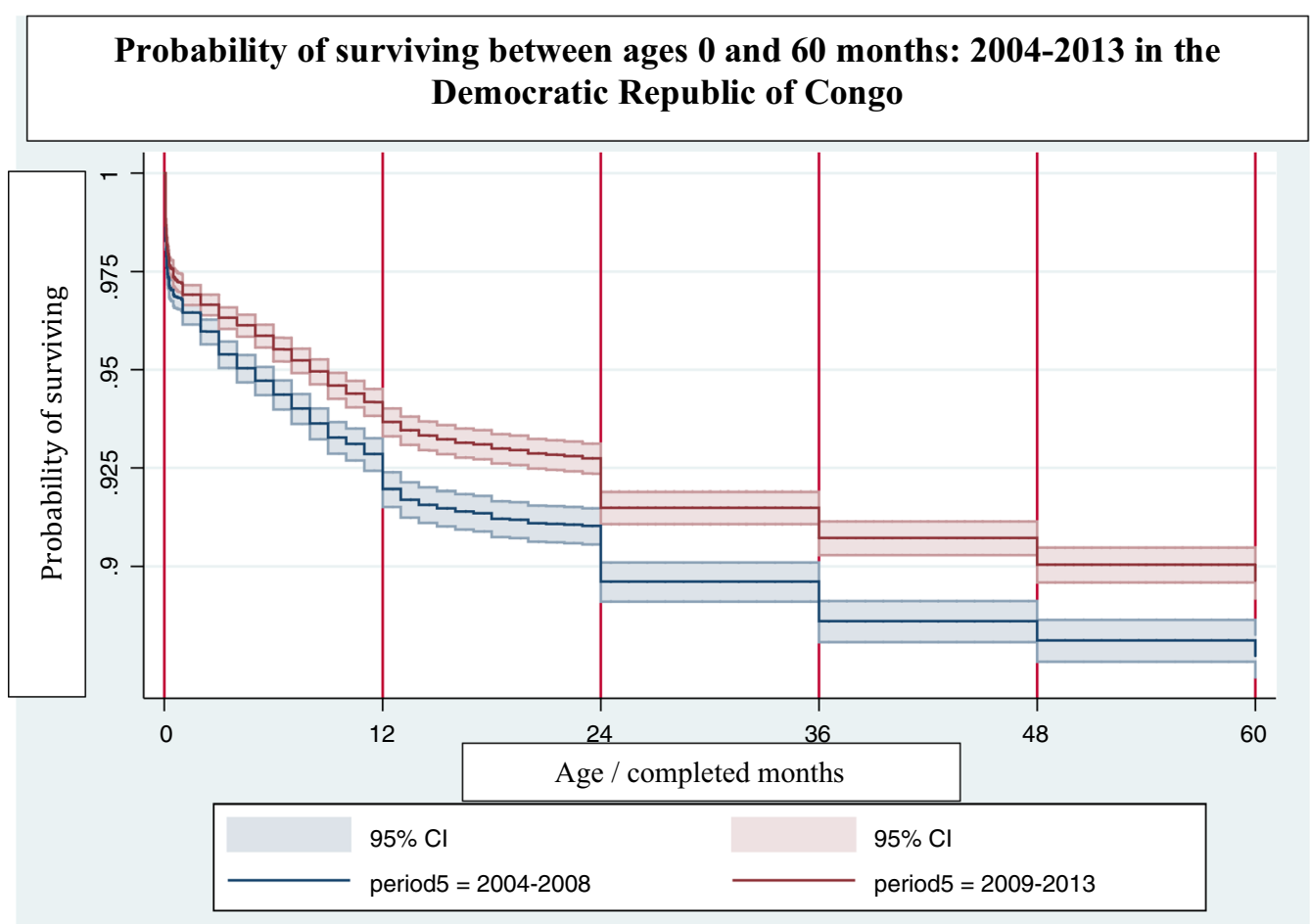

Fig. 8 Kaplan-Meier survival probability. Cl confidence interval

\section{Cox regression}

The Cox proportional hazards regression examined the risk of mortality among children aged 0-59 months in relation to household ownership of ITNs. Household ownership of at least one ITN reduced the risk of mortality among children aged $0-59$ months by 24 percentage points during the 24-month period before the survey (risk ratio $=0.76,95 \%$ CI 0.64-0.90) (Table 2).

\section{Modest change in contextual factors}

Trends in contextual factors such as socioeconomic, maternal and child health interventions, and meteorological factors, were examined $[19,20]$. Gross domestic product per capita increased, from 218 USD in 2005 to 497 USD in 2015; however, the overall health expenditure also increased, from 9 USD in 2005 to more than 19 USD in 2015 [8, 21]. Most indicators of living conditions and sanitation were not significantly different from 2007 to 2013, such as access to improved water source (46\% to $49 \%)$ and access to improved sanitation (15\% to $18 \%$ ) (Appendix). From 2007 to 2013, most indicators of maternal and child health in DRC did not significantly improve. Two indicators of antenatal care, which were relatively high in 2007, improved modestly in 2013, with one being statistically significant. The proportion of women who gave birth in a health facility increased, from $70.1 \%$ (95\% CI $69.2-71.0 \%)$ in 2007 to $79.9 \%$ (95\% CI 79.3-80.5\%) in 2013. The proportion of births assisted by skilled personnel increased, from $74.0 \%$ (95\% CI $70.0-78.0 \%$ ) in 2007 to $80.1 \%$ (95\% CI $77.6-82.7 \%$ ) in 2013. The nutritional status among children under five assessed through the proportion receiving vitamin A also increased, from $54.6 \%$ (95\% CI 50.4-58.8\%) in 2007 to $70.3 \%$ (95\% CI 67.9-72.6\%) in 2013 and a decrease in stunting from $45.5 \%$ (95\% CI 42.7-48.4) in 2007 to $42.4 \%$ (40.4-44.3\%) in 2013. Of the child health indicators examined, only two have seen an increase greater than 10 percentage points between 2007 and 2013 [22]. Full basic vaccination increased, from 30.6\% (95\% CI 25.9-35.2\%) in 2007 to $45.3 \%$ (95\% CI 42.0-48.6\%) in 2013, and exclusive breastfeeding increased, from $36.1 \%$ (95\% CI 33.0$35.2 \%$ ) in 2007 to 47.6 (95\% CI $45.5-49.8 \%$ ) in 2013.

In addition to these contextual factors, the total annual rainfall over the assessment period, calculated from the difference between the total annual precipitation for the year and total annual precipitation for the previous 5 years, has mostly deviated between -50 and $+50 \mathrm{~mm}$, except for 2006, which had higher excess rain of $188 \mathrm{~mm}$ [9]. The average temperatures also experienced positive and negative deviations between -0.5 and $0.5{ }^{\circ} \mathrm{C}$ during 
Table 2 Effect of household ITN ownership on 0-59-month mortality with the Cox regression model in DRC from 2011 to 2013

\begin{tabular}{|c|c|}
\hline Variables & Risk ratio $(95 \% \mathrm{Cl})$ \\
\hline \multicolumn{2}{|l|}{ Predictor } \\
\hline \multicolumn{2}{|l|}{ Household ITN ownership } \\
\hline Household without ITN & Reference \\
\hline Household owning at least 1 ITN & $0.76(0.64-0.90)$ \\
\hline \multicolumn{2}{|l|}{ Characteristics } \\
\hline \multicolumn{2}{|l|}{ Age of the child (in months) } \\
\hline$<6$ & Reference \\
\hline $6-11$ & $0.40(0.31-0.51)$ \\
\hline $12-23$ & $0.20(0.14-0.27)$ \\
\hline $24-35$ & $0.09(0.06-0.15)$ \\
\hline $36-47$ & $0.06(0.03-0.11)$ \\
\hline $47-59$ & $0.02(0.01-0.08)$ \\
\hline \multicolumn{2}{|l|}{ Sex of the child } \\
\hline Male & Reference \\
\hline Female & $0.97(0.82-1.14)$ \\
\hline \multicolumn{2}{|l|}{ Mother's education } \\
\hline No education & Reference \\
\hline Primary & $1.21(0.97-1.5)$ \\
\hline Secondary or higher & $1.13(0.87-1.46)$ \\
\hline \multicolumn{2}{|l|}{ Mother's marital status } \\
\hline Single & Reference \\
\hline Married & $0.72(0.46-1.11)$ \\
\hline Single/widow & $0.98(0.60-1.60)$ \\
\hline \multicolumn{2}{|l|}{ Household wealth quintiles } \\
\hline Lowest & Reference \\
\hline Second & $0.98(0.77-1.23)$ \\
\hline Middle & $1.02(0.81-1.30)$ \\
\hline Fourth & $0.91(0.69-1.20)$ \\
\hline Highest & $0.45(0.30-0.68)$ \\
\hline \multicolumn{2}{|l|}{ Place of residence } \\
\hline Rural & Reference \\
\hline Urban & $0.77(0.61-0.96)$ \\
\hline Person month & $367,405.1$ \\
\hline N (death) & $19,820(550)$ \\
\hline
\end{tabular}

Bold = statistically significant

the evaluation period. However, despite these differences, mean annual temperatures remained between 24 and $25{ }^{\circ} \mathrm{C}$, which are favourable temperatures for malaria transmission. The total annual rainfall and temperature remained consistent and conducive to malaria transmission throughout the evaluation period; this regularity provides an appropriate background to evaluate the other indicators.

\section{Discussion}

This study identified a significant decrease in severe anaemia and ACCM in DRC from 2007 to 2013-2014. This downward trend is most likely driven by household ownership of ITNs and use of ITNs among children under five. During this same period, there was a significant increase in financial investments by the country and its partners, focused on the scale-up of malaria control interventions, particularly vector control, from 18 million USD in 2007 to 175 million USD in 2015 [23, 24]. The NMCP implemented three ITN mass distribution campaigns across the country from 2009 to 2014 , with a plan to distribute $1-3$ ITNs per household or about 35 million ITNs total $[25,26]$. This strategy and investments led to the significant increase in household ITN ownership and use among children under five. DRC nearly achieved its ITN ownership coverage target of $80 \%$. ITN ownership and use targets are often difficult to achieve. In a study of 22 sub-Saharan African countries, only 4 achieved their household ITN ownership targets, and none achieved their ITN use among children under five targets [27].

The results show a clear pattern, which can be observed through the changes across the 11 provinces. The subnational analysis revealed an overall increase in ITN ownership and a significant decrease in ACCM and severe anaemia, but most importantly, some of the provinces with the largest improvements in coverage of ITN ownership and use also reported the largest decrease in ACCM. A finding reflected in a similar assessment showed a strong correlation between a high level of ITN use and the protective effect against malaria among children under five in DRC [28]. The geospatial differences in provinces may explain why some provinces with high intervention coverage experienced minimal change in either severe anaemia or ACCM. These spatial differences result in varying levels of access to health care, implementation of specific health programmes, and exposure to conflict, which may affect health outcomes of children under five [29]. Families fleeing areas of significant conflict in east DRC may have affected ACCM in those areas and contributed to the slight decreases in ACCM observed in neighbouring provinces such as Equateur, Katanga, and Sud-Kivu. Provinces without conflict and high intervention coverage, such as Bandundu, experienced the second largest decrease in ACCM.

The implementation of routine distribution of ITNs through antenatal clinics and EPI services, with a focus on younger children, may have resulted in the more pronounced decline in child mortality among 
children aged 6-23 months, compared to those aged 24-59 months. This is significant because children aged 6-23 months are more susceptible to malaria mortality [30]. Similar results were found by Dolan et al., who identified a $41 \%$ reduction in ACCM among children living in rural areas due to ITN distribution campaigns that targeted the highest risk areas for malaria in DRC [31]. Furthermore, this observation is consistent with other studies in sub-Saharan Africa that found an association between an increase in ITN ownership and a decline in ACCM and severe anaemia [32-34].

The results of the Kaplan-Meier survival probability analysis and Cox proportional hazards regressions both indicated an improved probability of survival among children under five post-intervention scale-up, compared to pre-intervention scale-up, which further supports the association between the increase in ITN coverage and decrease in ACCM. These methods have been used in similar studies assessing ITN effectiveness in reducing child mortality [35].

In addition to the scale-up of ITNs, the NMCP planned to implement several interventions during the evaluation period, however, the results were not as promising. The trends analysis revealed that access to ACT was still very low. In addition, low adherence to treatment policies and use of non-ACT were prevalent across the country [36]. Low access to ACT may have contributed to the increase in severe anaemia in SudKivu, and minimal changes in Katanga and Bandundu, despite high ITN ownership and use. The deployment of IRS across the country has been limited to one province due to funding, technical and logistical challenges [25]. The scale-up of IPTp across the country has not reached a comparable scale to ITNs [25]. A study conducted in 2013 found that only $20 \%$ of public health facilities in DRC were stocking sulfadoxine-pyrimethamine for IPTp [37]. The unmet goals for these interventions strengthen the plausible attribution of ITN ownership and ITN use to the observed decrease in ACCM.

To further investigate potential factors that may have contributed to the decline in ACCM, the contextual factor analysis indicated only a modest improvement in living conditions, sanitation, and maternal and child health indicators, such as nutritional status, vaccination status, exclusive breastfeeding, and access to water and improved toilets from 2007 to 2014 [2, 38, 39]. There were also no substantial changes in climate, rainfall, and temperature between 2005 and 2014 that may have affected the national mortality trend. Based on the analysis of these key factors known to affect malaria incidence, it is unlikely that these non-malaria contextual factors can explain a large proportion of the $30 \%$ reduction of ACCM $[40,41]$.

Overall, these results support the conclusion that the increase in household ITN ownership and increase in ITN use among children under five have contributed to the observed decrease in ACCM and severe anaemia from 2005 to 2014 in DRC. This study provides an assessment of the intervention coverage achieved and describes malaria-related morbidity and mortality rates up to 2014 in DRC. These results also provide baseline data that will be important for the measurement of the imminent progress that will be achieved at the end of the current 2016-2020 NMSP and may inform the decision-making of the NMCP and its partners as they transition to a new NMSP. Further research will be needed to fully understand how an increase in ITN ownership and use results in decreased ACCM and severe anaemia. However, DRC has made significant progress since 2005 in increasing intervention coverage and decreasing the burden of malaria on the population.

\section{Limitations of the study}

The use of secondary data from the DHS 2007 and DHS 2013-2014 limited this study to the variables that were collected in those surveys. No further primary data were collected. The difference in methodology between national surveys precluded the inclusion of the 2010 and 2017-2018 DRC Multiple Indicator Cluster Survey, which may have provided another data point to help elucidate the effects of DRC's malaria control intervention scale-up. Without data on malaria parasite prevalence from DHS 2007, the authors could not assess the parasitaemia trends. Other factors which can potentially affect ACCM, such as spatial differences in health programme implementation, climate differences across provinces, ongoing conflict, and scale-up of other disease interventions should be accounted for in future analysis [29, 42].

\section{Conclusion}

DRC has made significant gains in the coverage of and access to key malaria control interventions, such as ITNs. As DRC continues to focus its energy on scaling up malaria control strategies, the results are encouraging for future gains against the disease. The evidence in this study supports the conclusion that the scale-up of malaria control interventions substantially contributed to the observed decline in ACCM in DRC from 2005 to 2014, despite the high malaria prevalence in the country. When the next DHS survey is conducted in DRC, a similar analysis will be helpful to evaluate the achievements and impact of DRC's 2016-2020 NMSP. 


\section{Appendix}

See Tables 3, 4 and 5

Table 3 Changes in contextual factors associated with household factors in DRC from 2007 to 2014. Source: DHS 2007 and 2013-2014

\begin{tabular}{|c|c|c|c|c|c|}
\hline \multirow[t]{2}{*}{ Characteristics } & \multicolumn{2}{|l|}{2007 DHS } & \multicolumn{2}{|l|}{ 2013-2014 DHS } & \multirow{2}{*}{$\begin{array}{l}\text { Absolute } \\
\text { change } \\
2014-2007\end{array}$} \\
\hline & $(95 \% \mathrm{Cl})$ & $\mathrm{N}$ & $(95 \% \mathrm{Cl})$ & $\mathbf{N}$ & \\
\hline \multicolumn{6}{|l|}{ Household characteristics } \\
\hline Access to safe drinking water & $46.2(39.9-52.7)$ & 8886 & $48.7(44.2-53.3)$ & 18,171 & 2.5 \\
\hline Access to improved toilets & $15.6(12.9-19.6)$ & 8886 & $18.4(16.1-21.0)$ & 18,171 & 2.8 \\
\hline Household with flooring & $19.5(16.1-23.3)$ & 8886 & $17.6(15.2-20.3)$ & 18,171 & 1.8 \\
\hline Household with electricity & $15.3(12.3-18.8)$ & 8886 & $13.5(11.1-16.4)$ & 18,171 & 1.8 \\
\hline Household with telephone (fixed or mobile) & $31.3(30.3-32.3)$ & 8886 & $39.9(39.2-40.6)$ & 18,171 & 8.6 \\
\hline \multicolumn{6}{|l|}{ Socio-demographic characteristics } \\
\hline $\begin{array}{l}\text { Proportion of women aged 15-49 who have at least } \\
\text { reached primary education }\end{array}$ & $79.2(75.6-82.3)$ & 9995 & $84.6(82.6-86.3)$ & 18,827 & 5.4 \\
\hline Proportion of married 15-49 year old women (in union) & $66.3(64.1-68.3)$ & 9995 & $64.2(62.5-66.0)$ & 18,827 & 2.1 \\
\hline
\end{tabular}

Drinking water sources: tap, fountain, borehole, protected wells, rainwater, and bottled water. Toilets not shared by other households: flush toilet, improved latrine, latrine with slab, composting, according to DHS standards. Floor covering includes cement, tile, carpet, other modern material

Bold = statistically significant

Cl confidence interval, DHS demographic and health survey

Table 4 Changes in contextual factors associated with maternal health in DRC from 2007 to 2014. Source: DHS 2007 and 2013-2014

\begin{tabular}{|c|c|c|c|c|c|}
\hline \multirow[t]{2}{*}{ Characteristics } & \multicolumn{2}{|l|}{2007 DHS } & \multicolumn{2}{|l|}{ 2013-2014 DHS } & \multirow{2}{*}{$\begin{array}{l}\begin{array}{l}\text { Absolute } \\
\text { change } \\
\text { 2014-2007 }\end{array}\end{array}$} \\
\hline & $(95 \% \mathrm{Cl})$ & $\mathbf{N}$ & $(95 \% \mathrm{Cl})$ & $\mathbf{N}$ & \\
\hline \multicolumn{6}{|l|}{ Fertility risks } \\
\hline Single high-risk birth ${ }^{\mathrm{a}}$ & $36.4(35.4-37.4)$ & 9003 & $38.8(38.1-39.5)$ & 18,390 & 2.4 \\
\hline High-risk multiple-risk birth & $25.0(24.1-25.9)$ & 9003 & $24.8(24.2-25.4)$ & 18,390 & 0.2 \\
\hline Avoidable risk birth $^{\mathrm{b}}$ & $61.3(60.3-62.3)$ & 9003 & $63.6(62.9-64.3)$ & 18,390 & 2.3 \\
\hline Unavoidable risk birth ${ }^{c}$ & $15.9(14.5-16.7)$ & 9003 & $14.1(13.6-14.6)$ & 18,390 & 1.8 \\
\hline Birth at intervals -24 months & $7.3(6.5-7.9)$ & 9003 & $8.2(7.5-8.8)$ & 18,390 & 0.9 \\
\hline Birth order $>3$ & $22.8(21.9-23.7)$ & 9003 & $23.9(23.3-24.5)$ & 18,390 & 1.1 \\
\hline Mother of age $<18$ and $>34$ years old & $6.3(5.8-6.8)$ & 9003 & $6.7(6.3-7.1)$ & 18,390 & 0.4 \\
\hline \multicolumn{6}{|l|}{ Coverage in prenatal care } \\
\hline Prenatal consultation (ANC) & $46.7(45.4-48.0)$ & 5473 & $48.0(47.1-48.9)$ & 11,065 & 1.3 \\
\hline At least $2 T T$ doses during pregnancy & $38.6(35.6-41.5)$ & 5473 & $43.2(42.3-44.1)$ & 11,065 & 4.6 \\
\hline Childbirth in a health facility ${ }^{d}$ & $70.1(69.2-71.0)$ & 8999 & $79.9(79.3-80.5)$ & 18,390 & 9.8 \\
\hline Birth assisted by qualified staff & $74.0(70.0-78.0)$ & 8999 & $80.1(77.6-82.7)$ & 18,390 & 6.1 \\
\hline
\end{tabular}

\footnotetext{
Bold = statistically significant
}

TTTetanus toxoid vaccine, Cl confidence interval, DHS demographic and health survey

${ }^{a}$ High-risk birth is defined as a birth that occurred before 24 months of the previous birth, multiple delivery, birth order $<3$, or a mother's age is $<18$ years old or $>34$ years old

${ }^{\mathrm{b}}$ Avoidable risk birth is defined as a birth at an age of $<18$ years old or $>34$ years old, interval of $<24$ months between births or a birth order $>3$

c Unavoidable risk birth is defined as any childbirth $<18$ or $>34$ years old

${ }^{\mathrm{d}}$ Concerns childbirth in all public semi-public and private structures 
Table 5 Changes in contextual factors associated with child survival in DRC from 2007 to 2014. Source: DHS 2007 and $2013-2014$

\begin{tabular}{|c|c|c|c|c|c|}
\hline \multirow[t]{2}{*}{ Characteristics } & \multicolumn{2}{|l|}{2007 DHS } & \multicolumn{2}{|l|}{ 2013-2014 DHS } & \multirow{2}{*}{$\begin{array}{l}\text { Absolute } \\
\text { change } \\
2014-2007\end{array}$} \\
\hline & $(95 \% \mathrm{Cl})$ & $\mathbf{N}$ & $(95 \% \mathrm{Cl})$ & $\mathbf{N}$ & \\
\hline \multicolumn{6}{|l|}{ Vaccine coverage } \\
\hline $\mathrm{BCG}$ & $71.7(70.3-72.3)$ & 1632 & $83.5(81.1-85.7)$ & 3634 & 11.8 \\
\hline DTP3 & $45.0(43.9-46.2)$ & 1632 & $61.0(57.4-64.6)$ & 3634 & 16.0 \\
\hline Polio3 & $45.7(43.5-48.4)$ & 1632 & $65.5(62.2-68.6)$ & 3634 & 19.8 \\
\hline Measles & $62.9(60.5-65.2)$ & 1632 & $72.2(69.7-74.7)$ & 3634 & 9.3 \\
\hline Total vaccine coverage & $30.6(25.9-35.2)$ & 1585 & $45.3(42.0-48.6)$ & 3634 & 14.7 \\
\hline \multicolumn{6}{|c|}{ Micronutrient supplementation and nutritional status } \\
\hline Vitamin A & $54.6(50.4-58.8)$ & 1585 & $70.3(67.9-72.6)$ & 15,271 & 15.7 \\
\hline Delayed growth & $45.5(42.7-48.4)$ & 3631 & $42.4(40.4-44.3)$ & 8059 & -3.1 \\
\hline Underweight & $25.1(22.2-27.9)$ & 3631 & $22.4(20.8-24.0)$ & 8059 & -2.7 \\
\hline Emaciation & $10(8.0-12.1)$ & 3631 & $7.9(7.0-9.0)$ & 8059 & -2.1 \\
\hline Low birth weight $(<2500 \mathrm{~g})$ & $7.7(6.9-8.4)$ & 6104 & $7.1(6.3-7.8)$ & 13,922 & 0.6 \\
\hline Early initiation ( $\leq 1$ h of childbirth) & $48.1(46.7-49.4)$ & 5266 & $51.9(50.7-53.1)$ & 7168 & 3.8 \\
\hline \multicolumn{6}{|l|}{ Maternal breastfeeding } \\
\hline Exclusive breastfeeding (6 months) & $36.1(33.0-39.2)$ & 927 & $47.6(45.4-49.8)$ & 1935 & 11.5 \\
\hline $\begin{array}{l}\% \text { of } 6-9 \text { months of breastfeeding and } \\
\text { complementary food }\end{array}$ & $82.2(79.0-85.4)$ & 555 & $78.7(75.5-81.0)$ & 1209 & 3.5 \\
\hline \multicolumn{6}{|c|}{ Coverage of integrated management of 'child's disease' } \\
\hline Diarrhea rehydration therapy & $44.9(42.2-47.6)$ & 1287 & $41.9(38.9-44.9)$ & 2818 & 3.0 \\
\hline Oral rehydration salts for diarrhea & $30.8(28.2-33.3)$ & 1287 & $39.2(36.4-42.2)$ & 2818 & 8.3 \\
\hline \multicolumn{6}{|l|}{ Other childhood diseases } \\
\hline Prevalence of diarrhea & $16.4(15.6-17.2)$ & 7987 & $16.8(15.6-18.0)$ & 17,017 & 0.4 \\
\hline Prevalence of ARI symptoms & $15.4(14.6-16.2)$ & 7987 & $6.7(5.9-7.1)$ & 17,017 & 8.7 \\
\hline
\end{tabular}

Percentage of children aged 12-23 months with recommended vaccinations [this coverage takes into account vaccines against BCG (against tuberculosis), three doses of diphtheria, tetanus, pertussis (DTP3), three doses of oral polio vaccine (Polio3), and measles]; after 2007 the DTP vaccine were given pentavalent form with antigens against Hepatitis B and Haemophilus influenzae type B included

Bold = statistically significant

$A R I$ acute respiratory infections, $N$ weighted sample, $C /$ confidence interval, $D H S$ demographic and health survey

\section{Abbreviations}

ACCM: All-cause child mortality; ACT: Artemisinin-based combination therapy; Cl: Confidence interval; EPI: Expanded programme on immunization; DHS: Demographic and Health Survey; DRC: Democratic Republic of Congo; IPTp: Intermittent preventive treatment in pregnancy; ITN: Insecticide-treated net; NMCP: National Malaria Control Programme; NMSP: National malaria strategic plan.

\section{Acknowledgements}

The authors would like to acknowledge all the malaria stakeholders in DRC for their role in providing the data and documentation related to this study. A special thank you to Yung-Ting Bonnenfant and Mame Niang from the United States Centers for Disease Control, and the DRC Ministry of Health, including the NMCP, for their collaboration.

\section{Authors' contributions}

YY designed the evaluation, conceptualized the manuscript, and provided guidance for writing; KJ, IN, and AA drafted the manuscript and addressed all the revisions; $\mathrm{OK}, \mathrm{EM}, \mathrm{JL}, J \mathrm{~J}, \mathrm{AS}, \mathrm{MH}$, and $\mathrm{MN}$ contributed to the design and implementation of the evaluation and provided inputs to the manuscript; YTB reviewed the manuscript and provided inputs, all co-authors reviewed the content of the manuscript. All authors read and approved the final manuscript.

\section{Funding}

This work was made possible by the generous support of the American people through the United States Agency for International Development (USAID) and the U.S. President's Malaria Initiative (PMI) under the terms of the PMI Measure Malaria Associate Award No. 7200AA19LA00001. PMI Measure Malaria is implemented by the University of North Carolina at Chapel Hill, in partnership with ICF Macro, Inc.; Tulane University; John Snow, Inc.; and Palladium International, LLC. The contents do not necessarily reflect the views of USAID/ PMI or the United States Government.

\section{Availability of data and materials}

The data that support the findings of this study are available from The DHS Program upon reasonable request and with permission of The DHS Program.

\section{Declarations}

Ethics approval and consent to participate

No primary data were collected for this study; therefore, no further institutional review board approval was sought.

Consent for publication

Not applicable. 


\section{Competing interests}

The authors declare that they have no competing interests.

\section{Author details}

${ }^{1}$ PMI Measure Malaria, University of North Carolina at Chapel Hill, Chapel Hill, NC, USA. ${ }^{2}$ ICF, Rockville, MD, USA. ${ }^{3}$ National Malaria Control Programme, Ministry of Health, Kinshasa, Democratic Republic of Congo. ${ }^{4}$ University of Lubumbashi, Lubumbashi, Democratic Republic of Congo. ${ }^{5}$ University of Kisangani, Kisangani, Democratic Republic of Congo. ${ }^{6}$ University of Kinshasa, Kinshasa, Democratic Republic of Congo. ${ }^{7}$ Population and Health Research Institute, Kinshasa, Democratic Republic of Congo. ${ }^{8}$ President's Malaria Initiative/U.S. Agency for International Development, Washington, DC, USA.

Received: 14 December 2020 Accepted: 13 May 2021

Published online: 29 May 2021

\section{References}

1. Ministère du Plan, Macro International. Enquête Démographique et de Santé, République Démocratique du Congo 2007. Calverton: Kinshasa; 2008.

2. Ministère du Plan et Suivi de la Mise en oeuvre de la Révolution de la Modernité (MPSMRM), Ministère de la Santé Publique (MSP), ICF International. Enquête Démographique et de Santé en République Démocratique du Congo 2013-2014. Rockville: Kinshasa; 2014.

3. Programme National de Lutte contre le Paludisme. Plan stratégique national de lutte contre le paludisme 2013-2015. Kinshasa; 2013.

4. Ntuku HMT. Malaria epidemiology and key control interventions in the Democratic Republic of Congo. Thesis, University of Basel; 2016.

5. Yé Y, Eisele TP, Eckert E, Korenromp E, Shah JA, Hershey CL, et al. Framework for evaluating the health impact of the scale-up of malaria control interventions on all-cause child mortality in sub-Saharan Africa. Am J Trop Med Hyg. 2017;97:9-19.

6. Hershey CL, Florey LS, Ali D, Bennett A, Luhanga M, Mathanga DP, et al. Malaria control interventions contributed to declines in malaria parasitemia, severe anemia, and all-cause mortality in children less than 5 years of age in Malawi, 2000-2010. Am J Trop Med Hyg. 2017;97:76-88.

7. Programme National de Lutte contre le Paludisme. Plan stratégique national de lutte contre le paludisme 2016-2020. Kinshasa; 2016.

8. The World Bank. GDP per capita. https://data.worldbank.org/indicator/SP. DYN.IMRT.IN.

9. The World Bank. Climate change knowledge portal. https://climatekno wledgeportal.worldbank.org/.

10. MEASURE Evaluation. Guidance for evaluating the impact of national malaria control programs in highly endemic countries. Rockville: Kinshasa; 2014.

11. Hershey CL, Bhattarai A, Florey LS, McElroy PD, Nielsen CF, Yé Y, et al. Implementing impact evaluations of malaria control interventions: process, lessons learned, and recommendations. Am J Trop Med Hyg. 2017:97:20-31.

12. Victora CG, Schellenberg JA, Huicho L, Amaral J, El Arifeen S, Pariyo G, et al. Context matters: interpreting impact findings in child survival evaluations. Health Policy Plan. 2005;20(Suppl 1):i18-31.

13. Habicht JP, Victora CG, Vaughan JP. Evaluation designs for adequacy, plausibility and probability of public health programme performance and impact. Int J Epidemiol. 1999;28:10-8.

14. Korenromp EL, Armstrong-Schellenberg JR, Williams BG, Nahlen BL, Snow RW. Impact of malaria control on childhood anaemia in Africa-a quantitative review. Trop Med Int Health. 2004;9:1050-65.

15. MEASURE DHS, President's Malaria Initiative, Roll Back Malaria Partnership, UNICEF, WHO household survey indicators for malaria control. Rockville: Kinshasa; 2018.

16. Cox DR. Analysis of survival data. New York: Chapman and Hall; 1984.

17. Cox DR. The statistical analysis of dependencies in point processes. In: Symposium on point processes. New York: Wiley; 1972.

18. Blossfeld H, Hamerle A, Mayer K. Event History analysis. Statistical theory and application in the social sciences. Hillsdale: Lawrence Erlbaum Associates; 1989.

19. IGME estimation methods used by the UN Inter-Agency Group for Child Mortality Estimation. https://childmortality.org/methods.

20. IHME. Infant and child mortality estimates by Country 1970-2010. Seattle: Institute for Health Metrics and Evaluation; 2010.
21. The World Bank. Current health expenditure per capita-Democratic Republic of Congo. https://data.worldbank.org/indicator/SH.XPD.CHEX. PC.CD?end $=2015$ \&locations $=C D \&$ start $=2005$.

22. WHO. World malaria report 2017. Geneva: World Health Organization; 2017.

23. WHO. World malaria report 2008. Geneva: World Health Organization; 2008.

24. WHO. World malaria report 2016. Geneva: World Health Organization; 2016.

25. PMI. Democratic Republic of Congo: malaria operational play FY 2014. PMl; 2014.

26. Programme National de Lutte contre le Paludisme. Plan stratégique national de lutte contre le paludisme 2007-2011. Kinshasa; 2007.

27. Andrada A, Herrera S, Ye Y. Are new national malaria strategic plans informed by the previous ones? A comprehensive assessment of subSaharan African countries from 2001 to present. Malar J. 2019;18:253.

28. Levitz L, Janko M, Mwandagalirwa K, Thwai KL, Likwela JL, Tshefu AK, et al. Effect of individual and community-level bed net usage on malaria prevalence among under-fives in the Democratic Republic of Congo. Malar J. 2018;17:39.

29. Kandala N-B, Mandungu TP, Mbela K, Nzita KPD, Kalambayi BB, Kayembe KP, et al. Child mortality in the Democratic Republic of Congo: cross-sectional evidence of the effect of geographic location and prolonged conflict from a national household survey. BMC Public Health. 2014;14:266.

30. Smithson P, Florey L, Salgado SR, Hershey CL, Masanja H, Bhattarai A, et al. Impact of malaria control on mortality and anemia among Tanzanian children less than five years of age, 1999-2010. PLoS ONE. 2015;10:e0141112.

31. Dolan CB, BenYishay A, Grépin KA, Tanner JC, Kimmel AD, Wheeler DC, et al. The impact of an insecticide treated bednet campaign on all-cause child mortality: a geospatial impact evaluation from the Democratic Republic of Congo. PLoS ONE. 2019;14:e0212890.

32. Florey LS, Bennett A, Hershey CL, Bhattarai A, Nielsen CF, Ali D, et al. Impact of insecticide-treated net ownership on all-cause child mortality in Malawi, 2006-2010. Am J Trop Med Hyg. 2017;97:65-75.

33. Kayentao K, Florey LS, Mihigo J, Doumbia A, Diallo A, Kone D, et al. Impact evaluation of malaria control interventions on morbidity and all-cause child mortality in Mali, 2000-2012. Malar J. 2018;17:424.

34. Eckert E, Florey LS, Tongren JE, Salgado SR, Rukundo A, Habimana JP, et al. Impact evaluation of malaria control interventions on morbidity and all-cause child mortality in Rwanda, 2000-2010. Am J Trop Med Hyg. 2017;97:99-110.

35. Amek NO, Van Eijk A, Lindblade KA, Hamel M, Bayoh N, Gimnig J, et al. Infant and child mortality in relation to malaria transmission in KEMRI/CDC HDSS, Western Kenya: validation of verbal autopsy. Malar J. 2018;17:37.

36. Ntamabyaliro NY, Burri C, Nzolo DB, Engo AB, Lula YN, Mampunza SM, et al. Drug use in the management of uncomplicated malaria in public health facilities in the Democratic Republic of the Congo. Malar J. 2018;17:189.

37. Alum A, Andrada A, Archer J, Auko E, Bates K, Bouanchaud P, et al. The malaria testing and treatment market in Kinshasa, Democratic Republic of the Congo, 2013. Malar J. 2017;16:94.

38. Ngatu NR, Kanbara S, Renzaho A, Wumba R, Mbelambela EP, Muchanga SMJ, et al. Environmental and sociodemographic factors associated with household malaria burden in the Congo. Malar J. 2019;18:53.

39. United Nations. The Millenium development goals report. 2015. https:// www.un.org/millenniumgoals/2015_MDG_Report/pdf/MDG\%202015\% 20rev\%20(July\%201).pdf.

40. Kibret S, Glenn Wilson G, Ryder D, Tekie H, Petros B. Environmental and meteorological factors linked to malaria transmission around large dams at three ecological settings in Ethiopia. Malar J. 2019;18:54.

41. Rouamba T, Nakanabo-Diallo S, Derra K, Rouamba E, Kazienga A, Inoue Y, et al. Socioeconomic and environmental factors associated with malaria hotspots in the Nanoro demographic surveillance area, Burkina Faso. BMC Public Health. 2019;19:249.

42. Kandala NB, Emina JB, Nzita PD, Cappuccio FP. Diarrhoea, acute respiratory infection, and fever among children in the Democratic Republic of Congo. Soc Sci Med. 2009;68:1728-36.

\section{Publisher's Note}

Springer Nature remains neutral with regard to jurisdictional claims in published maps and institutional affiliations. 